\section{Characteristics of blood tests in patients with acute cerebral infarction who developed symptomatic intracranial hemorrhage after intravenous administration of recombinant tissue plasminogen activator}

\author{
Chungjo Lee', Ji Ung Na', Jang Hee Lee', Sang Kuk Han ${ }^{1}$, Pil Cho Choi ${ }^{1}$, \\ Young Hwan Lee ${ }^{2}$, Sang $O$ Park $^{3}$, Dong Hyuk Shin ${ }^{1}$ \\ 'Department of Emergency Medicine, Kangbuk Samsung Hospital, Sungkyunkwan University School of \\ Medicine, Seoul, Korea \\ 2Department of Emergency Medicine, Soonchunhyang University Bucheon Hospital, Bucheon, Korea \\ ${ }^{3}$ Department of Emergency Medicine, Konkuk University Medical Center, Konkuk University School of \\ Medicine, Seoul, Korea
}

Objective Patients suspected as having acute ischemic stroke usually undergo blood tests, including coagulation-related indexes, because thrombocytopenia and coagulopathy are contraindications for recombinant tissue plasminogen activator (rtPA) administration. We aimed to identify blood test indexes associated with symptomatic intracranial hemorrhage $(\mathrm{sICH})$ in patients with acute ischemic stroke who received intravenous rtPA.

Methods This retrospective observational study included patients diagnosed with acute ischemic stroke who were treated with intravenous rtPA at the emergency department of a tertiary hospital in Seoul between February 2008 and January 2018. Blood test indexes were compared between the $\mathrm{IICH}$ and non-sICH groups. Logistic regression and receiver-operating characteristic curve analyses were performed.

Results In this study, 375 patients were finally included. Of 375 patients, 42 (11.2\%) showed new intracranial hemorrhage on follow-up brain computed tomography, of whom 14 (3.73\%) had sICH. Platelet count, aspartate aminotransferase and lactate dehydrogenase levels were significantly different between the sICH and non-sICH groups, and platelet count showed statistical significance in the regression analysis. Significantly lower platelet counts were observed in the $\mathrm{sICH}$ group than in the non-sICH group $\left(174,500\right.$ vs. $\left.228,000 / \mathrm{mm}^{3}, \mathrm{P}=0.020\right)$. The best cutoff platelet count was $195,000 / \mathrm{mm}^{3}$, and patients with platelet counts of $<195,000 / \mathrm{mm}^{3}$ had a $5.4-$ times higher risk of developing $\mathrm{sICH}$ than those with platelet counts of $\geq 195,000 / \mathrm{mm}^{3}$.

Conclusion Platelet count was the only independent parameter associated with $\mathrm{sICH}$ among the blood test indexes. Mild thrombocytopenia may increase the risk of $\mathrm{sICH}$ after intravenous administration of rtPA.

Keywords Cerebral infarction; Thrombolytic therapy; Intracranial hemorrhages; Hematologic tests
elSSN: 2383-4625

Received: 12 July 2018

Revised: 14 August 2018

Accepted: 24 August 2018

Correspondence to: Dong Hyuk Shin Department of Emergency Medicine, Kangbuk Samsung Hospital,

Sungkyunkwan University School of Medicine, 29 Saemunan-ro, Jongnogu, Seoul 03181, Korea

E-mail: donghyuk.shinn@samsung.com ORCID

http://orcid.org/0000-0002-3226-2510

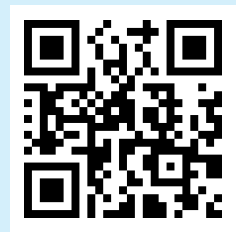

How to cite this article:

Lee C, Na JU, Lee JH, Han SK, Choi PC, Lee YH, Park SO, Shin DH. Characteristics of blood tests in patients with acute cerebral infarction who developed symptomatic intracranial hemorrhage after intravenous administration of recombinant tissue plasminogen activator. Clin Exp Emerg Med 2019;6(2):160-168.

This is an Open Access article distributed under the terms of the Creative Commons Attribution Non-Commercial License (http:// creativecommons.org/licenses/by-nc/4.0/). 


Capsule
Wummary is already known
$\begin{aligned} & \text { Previous studies reported that hyperglycemia, increased neutrophil-to-lymphocyte ratio, and decreased glomerular fil- } \\ & \text { tration rate are associated with the occurrence of symptomatic intracranial hemorrhage }(s / \mathrm{CH}) \text { after use of intravenous } \\ & \text { thrombolytic agents in patients with acute ischemic stroke. }\end{aligned}$
$\begin{aligned} & \text { What is new in the current study } \\ & \text { Platelet count was the only independent parameter associated with the development of } s / \mathrm{CH} \text {. Mild thrombocytopenia } \\ & \text { may increase the risk of sICH after intravenous administratino of thrombolytic agents. }\end{aligned}$

\section{INTRODUCTION}

Since 2010, the American Heart Association (AHA) and American Stroke Association (ASA) have recommended that recombinant tissue plasminogen activator (rtPA) be administered to patients within 4.5 hours of acute ischemic stroke onset. ${ }^{1}$ Although intravenous (IV) rtPA is an important and effective medical therapy that improves the prognosis of patients with an acute ischemic stroke, $^{2-5}$ hemorrhagic transformation occurs more frequently in the rtPA-treated group than in the placebo group. ${ }^{2,3,5,6}$ Hemorrhagic transformation is divided into petechial hemorrhage (scattered or spotty distribution) and parenchymal hemorrhage (hematoma) according to the configuration of hemorrhage on brain computed tomography (CT). ${ }^{3}$ Small petechial hemorrhages in infarcted tissue are not associated with poor prognosis; however, large parenchymal hematomas are associated with delayed recanalization and often result in neurological deterioration and poor outcome (symptomatic intracranial hemorrhage, sICH). ${ }^{7} \mathrm{Al}-$ though $\mathrm{sICH}$ is defined slightly differently among previous studies, $\mathrm{sICH}$ occurred in $1.7 \%$ to $7.6 \%$ of patients treated with rtPA. ${ }^{2-5,8}$ The incidence of large intracranial hemorrhage (parenchymal hemorrhage type 2) reported in a recent pooled analysis study was $5.2 \% .^{9}$

Patients suspected as having acute ischemic stroke usually undergo blood tests, including coagulation-related indexes such as platelet count, activated partial thromboplastin time (aPTT), and international normalized ratio of prothrombin time, because thrombocytopenia (platelet count $<100,000 / \mathrm{mm}^{3}$ ) and coagulopathy (international normalized ratio $>1.7$, abnormal aPTT) are contraindications for rtPA administration. ${ }^{10}$ However, considering that time is critical in patients with acute ischemic stroke, rtPA is sometimes administered before blood test results are obtained. ${ }^{10}$ Therefore, clinicians may face a dilemma of choosing between "rapid rtPA administration" and "confirming laboratory contraindications for rtPA" in the treatment decision of acute ischemic stroke. Naturally, coagulation-related blood test indexes may be associated with sICH occurrence. However, platelet count and aPTT were not included as risk factors of sICH. ${ }^{11}$ In contrast, non-coagulationrelated blood test indexes, such as hyperglycemia, ${ }^{11-13}$ increased neutrophil-to-lymphocyte ratio (NLR) ${ }_{1}^{14}$ and decreased glomerular filtration rate $(\mathrm{GFR}){ }_{1}^{15}$ were correlated with $\mathrm{sICH}$.

This study began with the question of whether the blood test indexes included in the contraindications for rtPA are associated with $\mathrm{SICH}$. This study aimed to investigate blood test indexes associated with sICH in Korean patients who had an acute ischemic stroke that was treated with rtPA and determine whether hematological indexes could predict sICH occurrence.

\section{METHODS}

\section{Study design and subjects}

This retrospective observational study included patients diagnosed with acute ischemic stroke and treated with IV rtPA at the emergency department (ED) of a tertiary hospital in Seoul between February 2008 and January 2018. An average of 34,000 patients visit the ED annually. Approximately 380 patients were diagnosed as having acute ischemic stroke per year during the study period.

Adult patients diagnosed with acute ischemic stroke and receiving IV rtPA in the ED were identified. The exclusion criteria were a pre-stroke modified Rankin Scale score $>1$, rtPA administration $>4.5$ hours after symptom onset, and no follow-up brain imaging within 36 hours after rtPA administration. This study was conducted according to the research ethics guidelines of our hospital after obtaining institutional review board approval (2015-12-041). Written informed consent was exempted by the institutional review board. To protect the patients' personal information, the patient name, hospital number, date of birth, and social security number were deleted after assigning a serial number to each patient. 


\section{Intravenous thrombolytic therapy}

In the ED where this study was performed, the so-called "stroke fast track protocol" was applied consistently for patients suspected as having an acute ischemic stroke within 6 hours of symptom onset to prevent treatment delay regardless of the patient's ED arrival order. If the stroke fast track protocol is applied, an on-call physician (emergency medicine resident or neurology resident) is immediately called from the triage. After a brief, focused medical history taking and neurological examination by the physician, immediate blood tests and brain imaging (CT or magnetic resonance imaging) are performed according to the physician's order. If IV administration of rtPA is indicated, $10 \%$ of the $0.9-\mathrm{mg} / \mathrm{kg}$ dose of rtPA (Actilyse, Boehringer-Ingelheim, Ingelheim, Germany) is administered as a bolus, and the remaining $90 \%$ of the dose is administered over 60 minutes. All patients who received rtPA were admitted to the stroke care unit, and follow-up brain non-contrast CT was routinely performed within 24 to 36 hours. All scans were performed using a 4th-generation CT scanner, with a 5-mmthick slice.

\section{Data collection}

Data of all the patients were collected by reviewing the electronic medical records and order communication system. The data collected and analyzed for the study were age; sex; medical history; initial vital signs; initial National Institutes of Health Stroke Scale (NIHSS) score; intravenous administration of rtPA; results of the initial blood test at the ED, including complete blood count; prothrombin time (PT); aPTT; levels of glucose, aspartate aminotransferase (AST), alanine amino transferase, total bilirubin, blood urea nitrogen, creatinine, creatinine phosphokinase, lactate dehydrogenase (LDH), electrolyte, and C-reactive protein; initial brain imaging (brain $\mathrm{CT}$ or magnetic resonance imaging); and follow-up brain CT.

Hemorrhagic transformation was considered to occur when ICH was newly observed in the follow-up CT. Hemorrhagic transformation was further divided into 4 subtypes according to the definition of the European Cooperative Acute Stroke Study (ECASS) as follows: HI1, small petechial hemorrhage along the margins of the infarct; $\mathrm{HI}$, confluent petechiae within the infarcted area but no space-occupying effect; $\mathrm{PH} 1$, hematoma covering $\leq 30 \%$ of the infarcted area with some space-occupying effect; and PH2, hematoma covering $>30 \%$ of the infarcted area with substantial space-occupying effect. ${ }^{3}$ sICH was defined as "any hemorrhage with neurological deterioration concomitant with an NIHSS score of $\geq 4$ points or any hemorrhage leading to death" according to ECASS II. All brain images were reviewed by the emergency physician with reference to the radiologist's readings.

\section{Statistical analyses}

Statistical analyses were performed, first, to identify variables with statistically significant differences between the two groups, among hematologic, demographic, and clinical indexes. The MannWhitney U-test was used for continuous variables, and the chisquare test or Fisher exact test was used for nominal variables. Data were presented as medians and interquartile ranges. Further logistic regression analyses were performed for all laboratory and clinical parameters. Receiver-operating characteristic curve analysis was performed for continuous variables with statistical significance in the logistic regression analysis to analyze the predictability of sICH. Multivariate logistic regression analysis was performed for all variables with statistical significance in the logistic regression analysis. We used Stata ver. 13.0 (StataCorp., College Station, TX, USA) for our statistical analyses, and the statistical significance was based on a P-value $<0.05$.

\section{RESULTS}

During the study period, 420 patients received IV rtPA upon diagnosis of acute ischemic stroke in the ED. After excluding 45 patients, 375 patients were finally included. Ten patients were excluded because rtPA was administered after 4.5 hours from symptom onset. Twenty-seven patients who did not undergo followup brain CT after IV rtPA were also excluded, and another 8 patients were excluded because of a lack of electronic medical record data. Of 375 patients, 42 (11.2\%) had a new intracranial hemorrhage on follow-up brain CT. Twenty-eight patients (7.4\%) had hemorrhagic transformation without significant symptoms (non-sICH), and 14 (3.7\%) had significant symptoms (sICH). All the patients in the sICH group had a PH2 subtype (Fig. 1). Four patients in the non-sICH group had an HI1 subtype; 13, an HI2 subtype; 10, a PH1 subtype; and 1, a PH2 subtype (Fig. 1).

\section{Comparison of patient characteristics between the sICH and non-sICH groups}

The patients in the post-rtPA sICH group were older than those in the non-sICH group, but the difference was not statistically significant ( 74 vs. 70 years, $P=0.734$ ). The incidence of sICH was higher in patients taking aspirin than in those not taking aspirin, but the difference was not statistically significant (9.42\% vs. $21.43 \%$, $P=0.150$ ). Initial systolic blood pressure (SBP), NIHSS score, and prevalence of atrial fibrillation were significantly higher in the post-rtPA sICH group than in the non-sICH group (SBP: 175 vs. $152 \mathrm{mmHg}, \mathrm{P}=0.041$; NIHSS: 18 vs. $10, \mathrm{P}<0.001$; atrial fibrillation: $64.29 \%$ vs. $34.07 \%, P=0.02$ ). The demographic and clinical features of the two groups are summarized in Table 1. 
420 Acute ischemic strokes treated with intravenous rtPA 0-4.5 hours after stroke onset

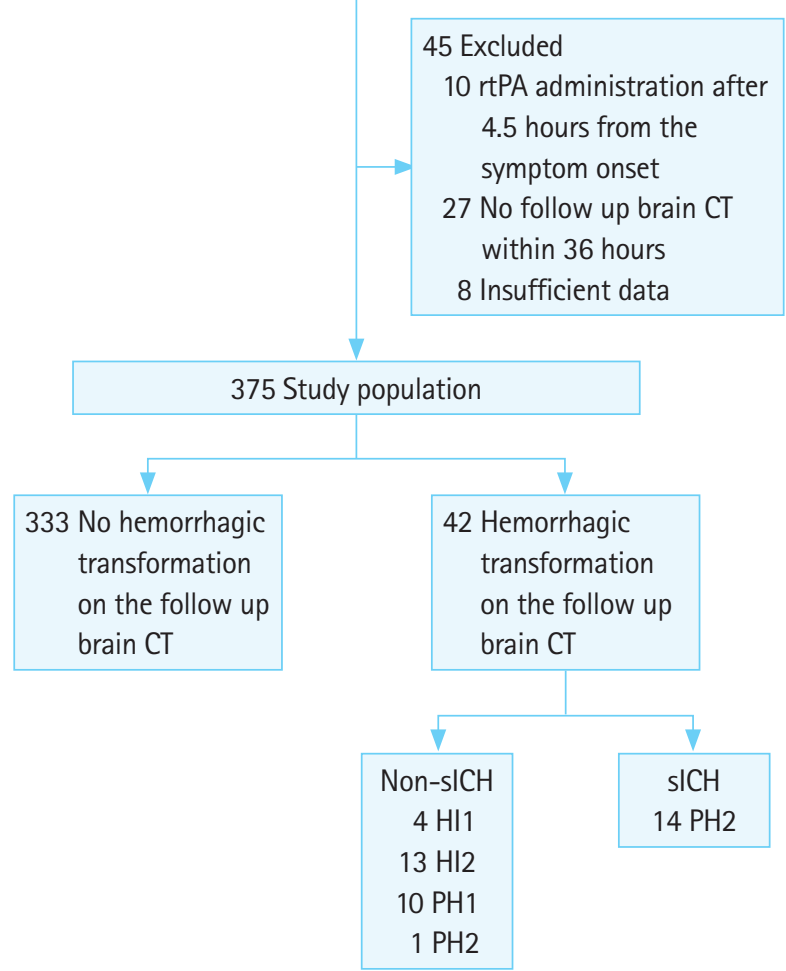

Fig. 1. Study flow diagram. rtPA, recombinant tissue plasminogen activator; $\mathrm{CT}$, computed tomography; $\mathrm{sICH}$, symptomatic intracranial hemorrhage; HI1, small petechial hemorrhage along the margins of the infarct; HI2, confluent petechiae within the infarcted area but no spaceoccupying effect; $\mathrm{PH} 1$, hematoma covering $\leq 30 \%$ of the infarcted area with some space-occupying effect; $\mathrm{PH} 2$, hematoma covering $>30 \%$ of the infarcted area with substantial space-occupying effect.

\section{Comparison of blood test indexes between the $\mathrm{sICH}$ and non-sICH groups}

Among the blood test indexes, platelet count, AST level, and LDH level were significantly different between the $\mathrm{sICH}$ and non-sICH groups. The platelet count was lower, and AST and LDH levels were higher in the sICH group than in the non-sICH group $(174,500$ vs. $228,000 / \mathrm{mm}^{3}, \mathrm{P}=0.02 ; 31$ vs. $24 \mathrm{IU} / \mathrm{L}, \mathrm{P}=0.022 ;$ and 296 vs. 395 IU/L, $P=0.05$, respectively). NLR, serum glucose level, and creatinine level were not statistically different between both the groups. The blood test indexes of both the groups are comparatively summarized in Table 2.

\section{Parameters to predict post-rtPA sICH}

In the univariate logistic regression analysis, SBP (odds ratio [OR], 1.018; 95\% confidence interval $[\mathrm{Cl}], 1.001$ to $1.034 ; \mathrm{P}=0.032$ ), NIHSS score $(\mathrm{OR}, 1.163 ; 95 \% \mathrm{Cl}, 1.061$ to $1.275 ; \mathrm{P}=0.001)$, presence of atrial fibrillation $(\mathrm{OR}, 3.483 ; 95 \% \mathrm{Cl}, 1.142$ to 10.618 ;
Table 1. Summary of the patients' characteristics

\begin{tabular}{|c|c|c|c|}
\hline Characteristics & $\begin{array}{l}\text { Non-sICH } \\
(n=361)\end{array}$ & sICH $(n=14)$ & P-value \\
\hline Age (yr) & $70(60-77)$ & $74(60-76)$ & 0.734 \\
\hline$\geq 80$ & $68(18.84)$ & $1(7.14)$ & 0.481 \\
\hline Male & $226(62.6)$ & $7(50)$ & 0.340 \\
\hline Body weight (kg) & $62(55-70)$ & $58.5(45-64)$ & 0.083 \\
\hline \multicolumn{4}{|l|}{ Clinical parameters (prior to rtPA) } \\
\hline \multicolumn{4}{|l|}{ Systolic blood pressure $(\mathrm{mmHg})$} \\
\hline Initial & $152(131-178)$ & $175(156-197)$ & $0.041^{\mathrm{a})}$ \\
\hline Immediately before rtPA & $150(130-162)$ & $161.5(138-177)$ & 0.126 \\
\hline \multicolumn{4}{|l|}{ Diastolic blood pressure $(\mathrm{mmHg})$} \\
\hline Initial & $84(74-93)$ & $92.5(78-101)$ & 0.269 \\
\hline Immediately before rtPA & $80(70-90)$ & $80.5(67-100)$ & 0.630 \\
\hline Heart rate (initial) (counts/min) & $80(70-91)$ & $82.5(76-88)$ & 0.489 \\
\hline NIHSS (initial) & $10(6-16)$ & $18(15-19)$ & $<0.001^{\text {a) }}$ \\
\hline Onset to rtPA time (min) & $108(85-147)$ & $106.5(90-132)$ & 0.895 \\
\hline eGFR $\left(\mathrm{mL} / \mathrm{min} / 1.73 \mathrm{~m}^{2}\right)$ & $80.7(68.1-101)$ & 75.75 & 0.394 \\
\hline \multicolumn{4}{|l|}{ History } \\
\hline Hypertension & $210(58.17)$ & $8(57.14)$ & 0.939 \\
\hline Diabetes mellitus & $80(22.16)$ & $3(21.43)$ & 1.000 \\
\hline Hypercholesterolemia & $32(8.86)$ & $0(0)$ & 0.619 \\
\hline Atrial fibrillation & $123(34.07)$ & 9 (64.29) & $0.020^{a)}$ \\
\hline Smoking & $87(24.1)$ & $1(7.14)$ & 0.203 \\
\hline Previous stroke & $48(13.3)$ & $1(7.14)$ & 1.000 \\
\hline Previous intracranial hemorrhage & $1(0.28)$ & $1(7.14)$ & 0.073 \\
\hline mRS 0-1 before stroke & $356(98.61)$ & $14(100)$ & 1.000 \\
\hline rtPA dose $(\mathrm{mg})$ & $55.8(49-63)$ & $51.5(40.5-58)$ & 0.067 \\
\hline Antithrombotic drugs & $86(23.82)$ & $4(28.57)$ & 0.750 \\
\hline Aspirin monotherapy & $34(9.42)$ & $3(21.43)$ & 0.150 \\
\hline Clopidogrel monotherapy & $14(3.88)$ & $0(0)$ & 1.00 \\
\hline Aspirin and clopidogrel & $17(4.71)$ & $1(7.14)$ & 0.504 \\
\hline Warfarin & $16(4.43)$ & $0(0)$ & 1.000 \\
\hline Apixaban & $1(0.28)$ & $0(0)$ & 1.000 \\
\hline Triflusal & $2(0.55)$ & $0(0)$ & 1.000 \\
\hline Cilostazol & $2(0.55)$ & $0(0)$ & 1.000 \\
\hline
\end{tabular}

Values are presented as median (interquartile range) or number (\%). The eGFR was calculated using the 4-variable Modification of Diet in Renal Disease formula: eGFR $\left(\mathrm{mL} / \mathrm{min} / 1.73 \mathrm{~m}^{2}\right)=186 \times\left(\right.$ serum creatinine $\left.\mathrm{e}^{-1.154}\right) \times$ age $^{-0.203} \times(1.210$ if black) $\times 0.742$ (if female).

sICH, symptomatic intracranial hemorrhage; rtPA, recombinant tissue plasminogen activator; NIHSS, National Institutes of Health Stroke Scale; eGFR, estimated glomerular filtration rate; $m R S$, modified Rankin Scale.

a) Statistical significance, according to a Mann-Whitney U-test.

$\mathrm{P}=0.028)$, and platelet count $(\mathrm{OR}, 0.988 ; 95 \% \mathrm{Cl}, 0.978$ to 0.998 ;

$\mathrm{P}=0.018$ ) showed statistically significant correlations with $\mathrm{sICH}$ development (Table 3).

In the receiver-operating characteristic analysis, SBP and platelet count showed poor predictability (area under the curve [AUC], $0.661 ; 95 \% \mathrm{Cl}, 0.510$ to $0.811 ; \mathrm{AUC}, 0.684 ; 95 \% \mathrm{Cl}, 0.503$ to 0.865 , respectively) for sICH. NIHSS score showed fair predictability for sICH (AUC, 0.780; 95\% Cl, 0.713 to 0.848). The best cutoff SBP was 
Table 2. Comparison of blood test indexes between the sICH and non-sICH groups

\begin{tabular}{|c|c|c|c|}
\hline Blood test index & Non-sICH $(n=361)$ & sICH $(n=14)$ & P-value \\
\hline $\operatorname{WBC}\left(\times 1,000 / \mathrm{mm}^{3}\right)$ & $7.8(6.42-9.5)$ & $7.8(6.2-9.6)$ & 0.859 \\
\hline Neutrophil $(\%)^{\mathrm{a})}$ & $56.1(47-64.3)$ & $55.55(40.1-71.3)$ & 0.898 \\
\hline Monocyte $(\%)^{c)}$ & $56.1(47-64.3)$ & $55.55(40.1-71.3)$ & 0.898 \\
\hline Hemoglobin ( $\mathrm{g} / \mathrm{dL}$ ) & $13.9(12.5-15.1)$ & $13(12.6-14.1)$ & 0.203 \\
\hline Hematocrit (\%) & $41.6(38-44.8)$ & $39.65(37.1-42.9)$ & 0.252 \\
\hline $\mathrm{MCH}(\mathrm{pg})$ & $31(30-32.1)$ & $31.4(30.4-32.1)$ & 0.551 \\
\hline $\mathrm{MCHC}(\mathrm{g} / \mathrm{dL})$ & $33.5(32.9-34.1)$ & $33.4(32.7-34)$ & 0.737 \\
\hline RDW (\%) & $13.3(12.8-13.9)$ & $13.55(13.2-14.1)$ & 0.145 \\
\hline Platelet $\left(\times 1,000 / \mathrm{mm}^{3}\right)$ & $228(190-269)$ & $174.5(140-251)$ & $0.020^{d)}$ \\
\hline NLR & $1.66(1.14-2.63)$ & $1.555(0.86-3.26)$ & 0.825 \\
\hline LMR & $0.6(0.38-0.88)$ & $0.65(0.31-1.16)$ & 0.813 \\
\hline Blood urea nitrogen (mg/dL) & $17.4(13.9-20.8)$ & $20.2(16.5-23.4)$ & 0.143 \\
\hline Creatinine $(\mathrm{mg} / \mathrm{dL})$ & $0.9(0.8-1.1)$ & $1(0.8-1.2)$ & 0.565 \\
\hline Aspartate transaminase (IU/L) & $24(19-28)$ & $31(23-38)$ & $0.022^{\mathrm{d})}$ \\
\hline Alanine aminotransferase (IU/L) & $19(14-25)$ & $22(13-30)$ & 0.551 \\
\hline Bilirubin (mg/dL) & $0.72(0.54-0.92)$ & $0.805(0.69-1.135)$ & 0.169 \\
\hline Sodium (mmol/L) & 140 (139-142) & $140(139-141)$ & 0.799 \\
\hline Potassium (mmol/L) & $4(3.7-4.2)$ & $3.85(3.8-4.3)$ & 0.986 \\
\hline Chloride (mmol/L) & $105(103-107)$ & $104(102-107)$ & 0.698 \\
\hline Glucose (mg/dL) & $130(111-161)$ & $136.5(118-170)$ & 0.403 \\
\hline Creatine phosphokinase (IU/L) & $87.5(62-123)$ & $79.5(65-106)$ & 0.530 \\
\hline
\end{tabular}

Values are presented as median (interquartile range).

sICH, symptomatic intracranial hemorrhage; WBC, white blood cell count; $M C V$, mean corpuscular volume; $M C H$, mean corpuscular hemoglobin; $M C H C$, mean corpuscular hemoglobin concentration; RDW, red blood cell distribution width; NLR, neutrophil-to-lymphocyte ratio; LMR, lymphocyte-to-monocyte ratio; PLR, platelet-to-lymphocyte ratio; PT, prothrombin time; INR, international normalized ratio; aPTT, activated partial thromboplastin time.

a)Proportion (\%) of neutrophils in the WBC. ${ }^{b)}$ Proportion (\%) of lymphocytes in the WBC. ${ }^{\mathrm{c}}$ Proportion (\%) of monocytes in the WBC. ${ }^{\text {d) }}$ Statistical significance, according to the Mann-Whitney test.

$170 \mathrm{mmHg}$, with a sensitivity, specificity, positive predictive value, and negative predictive value of $64.29 \%, 66.76 \%, 6.98 \%$, and $97.97 \%$, respectively. The best cutoff platelet count was 195,000/ $\mathrm{mm}^{3}$, with a sensitivity, specificity, positive predictive value, and negative predictive value of $71.43 \%, 73.13 \%, 9.35 \%$, and $98.51 \%$, respectively. The best cutoff NIHSS score was 15 points, with a sensitivity, specificity, positive predictive value, and negative predictive value of $78.57 \%, 69.53 \%, 9.09 \%$, and $98.82 \%$, respectively.

In the multivariate regression analysis, SBP $\geq 170 \mathrm{mmHg}$, NIHSS score $\geq 15$, and platelet count $<195,000 / \mathrm{mm}^{3}$ showed adjusted ORs ( $95 \% \mathrm{Cl})$ of 3.247 (0.998 to $10.571, \mathrm{P}=0.050), 7.020$ (1.816 to $27.134, P=0.005$ ), and 5.389 (1.491 to $19.481, P=0.010$ ) (Table 4), respectively. The probabilities of sICH according to SBP,
NIHSS score, and platelet count are displayed in Fig. 2 with their predictive margins.

\section{DISCUSSION}

In this study, we identified blood test indexes that were associated with $\mathrm{sICH}$ in patients with acute ischemic stroke who received IV rtPA. Platelet count was the only independent parameter associated with $\mathrm{sICH}$ among all the blood test indexes. In addition, mild thrombocytopenia could increase the risk of sICH after IV administration of rtPA.

Some blood test indexes, such as hyperglycemia, ${ }^{11-13}$ increased $\mathrm{NLR}_{1}{ }^{14}$ and decreased $\mathrm{GFR}_{1}{ }_{1}^{15}$ are associated with post-rtPA sICH. Surprisingly, coagulation-related blood test indexes, such as plate- 
Table 3. Univariate logistic regression analysis of clinical and laboratory parameters

\begin{tabular}{|c|c|c|}
\hline Variable & Unadjusted OR (95\% Cl) & P-value \\
\hline \multicolumn{3}{|l|}{ Clinical parameters } \\
\hline Age & 1.006 (0.964-1.049) & 0.784 \\
\hline Male & $0.597(0.205-1.740)$ & 0.345 \\
\hline Body weight & $0.958(0.918-1.001)$ & 0.055 \\
\hline Initial SBP & $1.018(1.001-1.034)$ & $0.032^{\mathrm{a})}$ \\
\hline Initial DBP & $1.015(0.985-1.046)$ & 0.332 \\
\hline NIHSS & $1.163(1.061-1.275)$ & $0.001^{\mathrm{a})}$ \\
\hline Onset to rtPA time & 0.998 (0.987-1.010) & 0.755 \\
\hline eGFR & 0.999 (0.981-1.018) & 0.941 \\
\hline Hypertension & $0.959(0.326-2.820)$ & 0.939 \\
\hline Diabetes mellitus & $0.958(0.261-3.517)$ & 0.948 \\
\hline Atrial fibrillation & $3.483(1.142-10.618)$ & $0.028^{\mathrm{a})}$ \\
\hline rtPA dose & $0.953(0.908-1.000)$ & 0.051 \\
\hline Aspirin monotherapy & $2.623(0.698-9.864)$ & 0.154 \\
\hline Aspirin and clopidogrel & $1.557(0.192-12.603)$ & 0.678 \\
\hline \multicolumn{3}{|l|}{ Laboratory parameters } \\
\hline White blood cell count & $0.946(0.751-1.193)$ & 0.639 \\
\hline Neutrophil & $1.000(0.959-1.042)$ & 0.985 \\
\hline Hemoglobin & $0.889(0.697-1.134)$ & 0.344 \\
\hline Hematocrit & $0.959(0.878-1.048)$ & 0.356 \\
\hline RDW & $1.092(0.831-1.435)$ & 0.526 \\
\hline Platelet & 0.988 (0.978-0.998) & $0.018^{\mathrm{a})}$ \\
\hline NLR & $1.036(0.806-1.331)$ & 0.784 \\
\hline PLR & 0.999 (0.990-1.008) & 0.793 \\
\hline PT (INR) & 2.047 (0.015-277.702) & 0.775 \\
\hline aPTT & $1.074(0.930-1.240)$ & 0.331 \\
\hline Glucose fasting & $1.002(0.995-1.009)$ & 0.636 \\
\hline Bilirubin total & $2.582(0.534-12.490)$ & 0.238 \\
\hline Aspartate transaminase & 1.009 (0.991-1.028) & 0.318 \\
\hline Alanine aminotransferase & $1.004(0.974-1.036)$ & 0.780 \\
\hline Blood urea nitrogen & $1.038(0.977-1.103)$ & 0.228 \\
\hline Creatinine & $0.805(0.213-3.044)$ & 0.749 \\
\hline Lactate dehydrogenase & $1.003(0.999-1.006)$ & 0.110 \\
\hline
\end{tabular}

$\mathrm{OR}$, odds ratio; $\mathrm{Cl}$, confidence interval; $\mathrm{SBP}$, systolic blood pressure; $\mathrm{DBP}$, diastolic blood pressure; NIHSS, National Institutes of Health Stroke Scale; rtPA, recombinant tissue plasminogen activator; eGFR, estimated glomerular filtration rate; RDW, red blood cell distribution width; NLR, neutrophil-to-lymphocyte ratio; $\mathrm{PLR}$, platelet-to-lymphocyte ratio; PT, prothrombin time; INR, international normalized ratio; aPT, activated partial thromboplastin time.

a)Statistical significance.

let count, PT, and aPT, are not related to post-rtPA sICH. Unlike the results of previous studies, our results showed that serum glucose level, NLR, and GFR were not significantly different between the sICH and non-sICH groups. Rather, platelet count was the only independent parameter that predicted $\mathrm{sICH}$ development. The best cutoff platelet count was $195,000 / \mathrm{mm}^{3}$. When we divided subjects into groups of $<195,000 / \mathrm{mm}^{3}$ and $\geq 195,000 / \mathrm{mm}^{3}$ according to the platelet count, the median platelet count in the $<195,000 / \mathrm{mm}^{3}$ group was $164,000 / \mathrm{mm}^{3}$ and $9.4 \%(10 / 107) \mathrm{had}$
Table 4. Multivariate logistic regression analysis of clinical and laboratory parameters

\begin{tabular}{lcc}
\hline Variable & Adjusted OR (95\% Cl) & P-value \\
\hline Initial SBP & $1.017(1.000-1.034)$ & 0.050 \\
Initial SBP $\geq 185$ vs. $<185 \mathrm{mmHg}^{\text {b) }}$ & $2.124(0.632-7.145)$ & 0.223 \\
Initial SBP $\geq 170$ vs. $<170 \mathrm{mmHg}^{\text {c) }}$ & $3.247(0.998-10.571)$ & 0.050 \\
NIHSS & $1.160(1.046-1.286)$ & 0.005 \\
NIHSS $\geq 15$ vs. $<15^{\mathrm{d})}$ & $7.020(1.816-27.134)$ & 0.005 \\
Atrial fibrillation & $2.032(0.618-6.680)$ & 0.243 \\
Platelet count & $0.992(0.982-1.002)$ & 0.117 \\
Platelet $<100,000 / \mathrm{mm}^{3}$ vs. $\geq 100,000 / \mathrm{mm}^{3}$ & $40.905(1.954-856.466)$ & 0.017 \\
Platelet $<195,000 / \mathrm{mm}^{3}$ vs. $\geq 195,000 / \mathrm{mm}^{3 e)}$ & $5.389(1.491-19.481)$ & 0.010
\end{tabular}

$\mathrm{OR}$, odds ratio; $\mathrm{Cl}$, confidence interval; $\mathrm{SBP}$, systolic blood pressure; NIHSS, National Institutes of Health Stroke Scale.

${ }^{a)}$ Adjusted for initial SBP, NIHSS, atrial fibrillation, platelet count, lactate dehy-

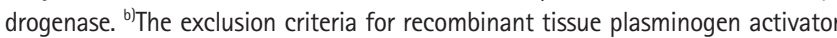
within 4.5 hours from stroke onset by the American Heart Association and the

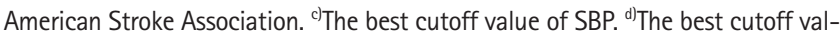
ue of the NIHSS score. ${ }^{\text {e}}$ The best cutoff value of platelet count.

sICH. The median platelet count was $250,500 / \mathrm{mm}^{3}$ and $1.5 \%$ (4/ 268) had sICH in the $>195,000 / \mathrm{mm}^{3}$ group. Patients with platelet counts of $<195,000 / \mathrm{mm}^{3}$ had a 5.4-times higher risk of developing sICH than those with platelet counts of $\geq 195,000 / \mathrm{mm}^{3}$.

Our main finding suggests that mild thrombocytopenia may not be a contraindication for rtPA as long as the platelet count is $>100,000 / \mathrm{mm}^{3}$ but may still increase the risk of post-rtPA sICH. This main result regarding platelet count may have significant clinical implications and may be controversial. In the AHA/ASA guidelines, rtPA is contraindicated for patients with platelet counts of $<100,000 / \mathrm{mm}^{3},{ }^{10}$ but in the real world, it may be administered before confirming platelet counts. Mowla et al. ${ }^{16}$ reported no significant difference in the incidence of post-rtPA sICH between patients with platelet counts of $<100,000 / \mathrm{mm}^{3}$ and those with platelet counts of $>100,000 / \mathrm{mm}^{3}(7.7 \%$ vs. $6.04 \%, P=0.73)$. However, their study included only a few cases (five cases plus 21 cases reported in previous studies), which are insufficient to make definitive conclusions. In our study, two patients had a platelet count of $<100,000 / \mathrm{mm}^{3}$, and sICH occurred in one (50\%). One patient who had sICH was a 75-year-old woman with an initial NIHSS score of 13 who received IV rtPA 71 minutes after symptom onset. Her platelet count was $87,000 / \mathrm{mm}^{3}$. The other patient was an 85-year-old man who did not have sICH but had an initial NIHSS score of 16. He received rtPA 90 minutes after symptom onset, and his platelet count was $99,000 / \mathrm{mm}^{3}$. In this study, patients with a platelet count $<100,000 / \mathrm{mm}^{3}$ showed a 40.9 -times higher risk of developing $\mathrm{sICH}$ than those with a platelet count $>100,000 / \mathrm{mm}^{3}$. Therefore, we recommend upholding the platelet count of $<100,000 / \mathrm{mm}^{3}$ as an absolute contraindication for IV 

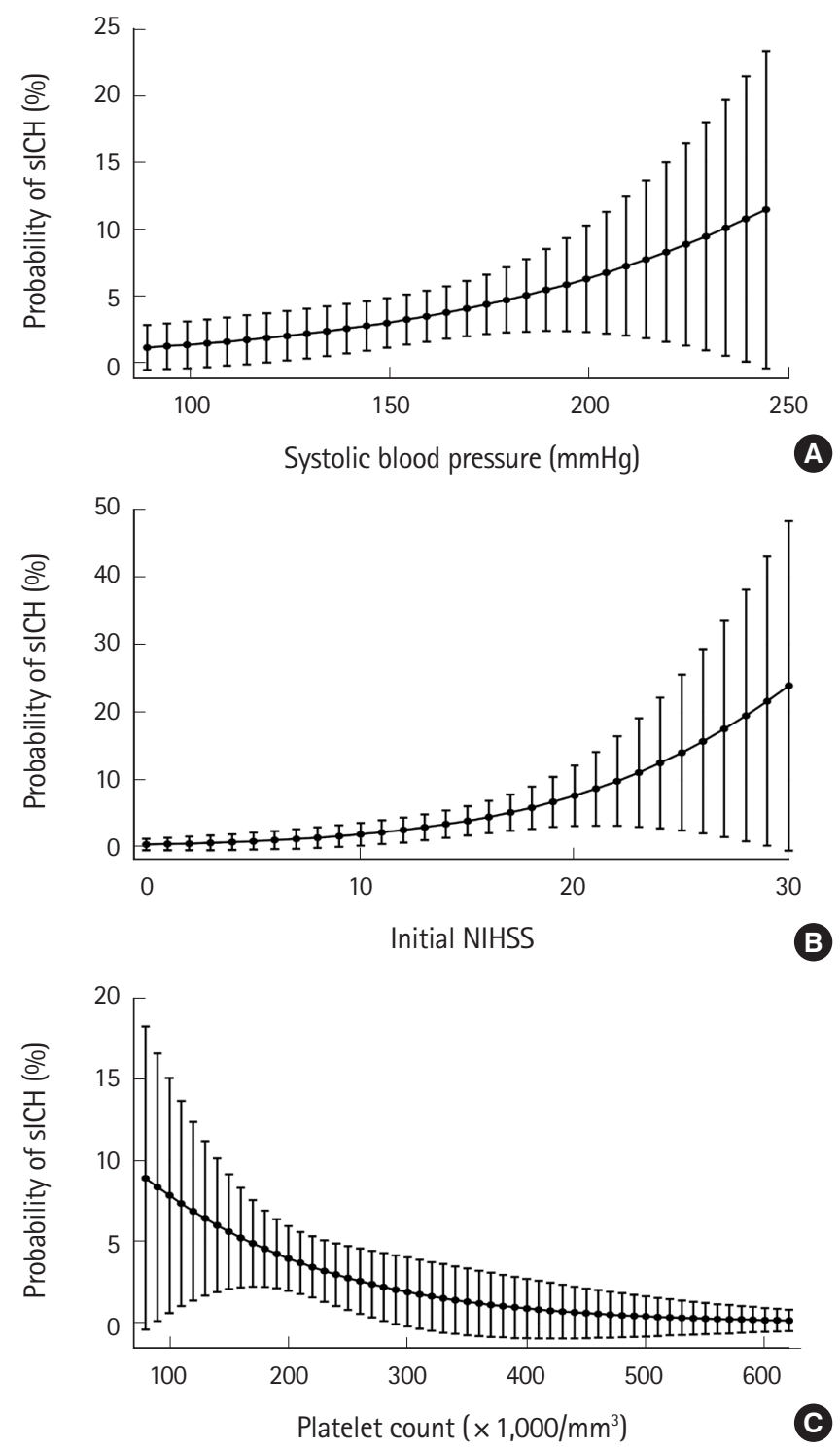

Fig. 2. Graphs of the probability of symptomatic intracranial hemorrhage ( $\mathrm{sICH}$ ) with predictive margins. Predictive probability of $\mathrm{sICH}$ according to systolic blood pressure (A), initial National Institutes of Health Stroke Scale (NIHSS) score (B), and platelet count (C).

rtPA administration.

In addition, the increased risk of sICH should not be ignored in cases within the lower normal range (mild thrombocytopenia). The 2018 AHA/ASA guidelines recommend intra-arterial thrombectomy for treating acute ischemic stroke caused by a documented large artery occlusion in the proximal anterior circulation. ${ }^{17}$ Second-generation mechanical thrombectomy devices (retrievable stents) are safe and effective for reducing disability and are superior to standard treatment with IV thrombolysis alone for treating acute ischemic stroke. Although it is beyond the scope of this study, it may be desirable to substitute endovascular throm- bectomy for IV or intra-arterial administration of rtPA in patients with low platelet counts, if possible.

Although AST and LDH levels were significantly different between the $\mathrm{IICH}$ and non-sICH groups, they may not be clinically related to sICH occurrence. In the logistic regression analysis, AST and LDH levels were statistically significant and therefore cannot be used as a predictor of post-rtPA sICH. However, we could not explain why AST and LDH levels were elevated in the sICH group.

Among the clinical risk factors, SBP and NIHSS score were independently associated with sICH in this study, and these results are consistent with those of previous studies. Perini et al. ${ }^{18}$ reported that higher SBP significantly increases the frequency of sICH in rtPA-treated patients. In the ECASS II study, the baseline SBP before rtPA administration showed a significant correlation with $\mathrm{sICH}(\mathrm{OR}, 1.02 ; 95 \% \mathrm{Cl}, 1.00$ to $1.03, \mathrm{P}=0.02) .^{12}$ The results of this study also showed that a higher initial SBP was independently associated with sICH among patients who received IV rtPA. Interestingly, the initial SBP at the ED was more strongly associated with the incidence of sICH than SBP just before administering IV rtPA. The best cutoff initial SBP for an increased risk of sICH was $170 \mathrm{mmHg}$. Although high blood pressure may theoretically be advantageous to improve regional cerebral blood flow ${ }_{1}^{19}$ it may be desirable to maintain SBP at $<170 \mathrm{mmHg}$ to prevent sICH occurrence.

NIHSS score correlates with infarct volume and is the most commonly used stroke outcome scale. ${ }^{20}$ In previous studies, higher initial NIHSS scores were associated with $\mathrm{sICH}_{1}{ }^{11,21}$ and similar results were obtained in this study. In this study, NIHSS score turned out to be the most reliable predictor of sICH among the other parameters assessed. Patients with NIHSS scores $\geq 15$ had a 7 -times higher risk of sICH than those with NIHSS scores $<15$. If a patient with an acute stroke has an NIHSS score $\geq 15$, the physician must be cautious in using rtPA and should check the patient's platelet count and strictly control SBP.

Previous studies do not agree on the association between age and $\mathrm{sICH}$. In the meta-analysis by Whiteley et al., ${ }^{11}$ age was associated with post-rtPA sICH $(\mathrm{OR}, 1.03 ; 95 \% \mathrm{Cl}, 1.01$ to $1.04, \mathrm{P}<0.001)$. However, in the prospective cohort study by Sylaja et al. ${ }^{22}$ older age in carefully selected patients did not increase the risk of postrtPA sICH. In the current study, 69 patients (18.4\%) were aged $>80$ years, and age and $\mathrm{sICH}$ were not statistically correlated.

This study has some limitations. First, it was conducted at a single tertiary hospital. As the research hospital is located in an urban area and the study was conducted by Asian people, care should be taken in comparing the results with those of previous studies because regional and racial characteristics may act as sources of bias. Second, although we analyzed the data for 10 
years, the number of sICH patients was only 14 . Nevertheless, the sample size of this study was relatively larger than those of other single-center studies. However, the small sample size is one of the major weaknesses of this study. Third, this study did not include all possible clinical and brain imaging findings, such as infarction volume, hyperdense cerebral artery sign, and presence of visible hypodensity, owing to the natural limitation of retrospective studies. This study merely analyzed the blood test results of patients with sICH on their follow-up brain CT. However, the primary goal of this study was to determine the laboratory features of patients with $\mathrm{sICH}$, so this limitation did not significantly affect the main outcome of this study. This study is meaningful in that it analyzed the association between $\mathrm{SICH}$ and various laboratory parameters that have not been well-studied previously.

In conclusion, among the blood tests indexes, platelet count was the only independent predictor of $\mathrm{sICH}$ after administering rtPA within 4.5 hours from the onset of acute ischemic stroke symptoms. Physicians should be aware of the increased risk of sICH in patients with mild thrombocytopenia. If patients with mild thrombocytopenia have other concomitant risk factors, such as an NIHSS score $\geq 15$ or SBP $\geq 170 \mathrm{mmHg}$, physicians should be aware of the potential risk of post-rtPA sICH and should strictly control the blood pressure.

\section{CONFLICT OF INTEREST}

No author has any conflict of interest.

\section{REFERENCES}

1. Powers WJ, Derdeyn CP, Biller J, et al. 2015 American Heart Association/American Stroke Association focused update of the 2013 guidelines for the early management of patients with acute ischemic stroke regarding endovascular treatment: a guideline for healthcare professionals from the American Heart Association/American Stroke Association. Stroke 2015; 46:3020-35.

2. National Institute of Neurological Disorders and Stroke rt-PA Stroke Study Group. Tissue plasminogen activator for acute ischemic stroke. N Engl J Med 1995;333:1581-7.

3. Hacke $W$, Kaste $M$, Fieschi $C$, et al. Randomised double-blind placebo-controlled trial of thrombolytic therapy with intravenous alteplase in acute ischaemic stroke (ECASS II). Second European-Australasian Acute Stroke Study Investigators. Lancet 1998;352:1245-51.

4. Wahlgren N, Ahmed N, Davalos A, et al. Thrombolysis with alteplase for acute ischaemic stroke in the Safe Implementa- tion of Thrombolysis in Stroke-Monitoring Study (SITS-MOST): an observational study. Lancet 2007;369:275-82.

5. Hacke W, Kaste M, Bluhmki E, et al. Thrombolysis with alteplase 3 to 4.5 hours after acute ischemic stroke. N Engl J Med 2008; 359:1317-29.

6. Clark WM, Wissman S, Albers GW, Jhamandas JH, Madden KP, Hamilton S. Recombinant tissue-type plasminogen activator (Alteplase) for ischemic stroke 3 to 5 hours after symptom onset. The ATLANTIS Study: a randomized controlled trial. Alteplase Thrombolysis for Acute Noninterventional Therapy in Ischemic Stroke. JAMA 1999;282:2019-26.

7. Molina CA, Alvarez-Sabin J, Montaner J, et al. Thrombolysisrelated hemorrhagic infarction: a marker of early reperfusion, reduced infarct size, and improved outcome in patients with proximal middle cerebral artery occlusion. Stroke 2002;33: 1551-6.

8. Shobha N, Buchan AM, Hill MD; Canadian Alteplase for Stroke Effectiveness Study (CASES). Thrombolysis at 3-4.5 hours after acute ischemic stroke onset: evidence from the Canadian Alteplase for Stroke Effectiveness Study (CASES) registry. Cerebrovasc Dis 2011;31:223-8.

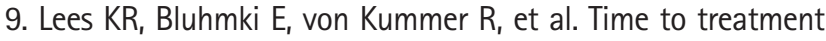
with intravenous alteplase and outcome in stroke: an updated pooled analysis of ECASS, ATLANTIS, NINDS, and EPITHET trials. Lancet 2010;375:1695-703.

10. Jauch EC, Saver JL, Adams HP Jr, et al. Guidelines for the early management of patients with acute ischemic stroke: a guideline for healthcare professionals from the American Heart Association/American Stroke Association. Stroke 2013;44:870947.

11. Whiteley WN, Slot KB, Fernandes P, Sandercock P, Wardlaw J. Risk factors for intracranial hemorrhage in acute ischemic stroke patients treated with recombinant tissue plasminogen activator: a systematic review and meta-analysis of 55 studies. Stroke 2012;43:2904-9.

12. Larrue V, von Kummer R R, Muller A, Bluhmki E. Risk factors for severe hemorrhagic transformation in ischemic stroke patients treated with recombinant tissue plasminogen activator: a secondary analysis of the European-Australasian Acute Stroke Study (ECASS II). Stroke 2001;32:438-41.

13. Marti-Fabregas J, Bravo Y, Cocho D, et al. Frequency and predictors of symptomatic intracerebral hemorrhage in patients with ischemic stroke treated with recombinant tissue plasminogen activator outside clinical trials. Cerebrovasc Dis 2007; 23:85-90.

14. Maestrini I, Strbian D, Gautier S, et al. Higher neutrophil counts before thrombolysis for cerebral ischemia predict worse out- 
comes. Neurology 2015;85:1408-16.

15. Gensicke $H$, Zinkstok SM, Roos YB, et al. IV thrombolysis and renal function. Neurology 2013;81:1780-8.

16. Mowla A, Kamal H, Lail NS, et al. Intravenous thrombolysis for acute ischemic stroke in patients with thrombocytopenia. J Stroke Cerebrovasc Dis 2017;26:1414-8.

17. Powers WJ, Rabinstein AA, Ackerson T, et al. 2018 guidelines for the early management of patients with acute ischemic stroke: a guideline for healthcare professionals from the American Heart Association/American Stroke Association. Stroke 2018;49:e46-110.

18. Perini F, De Boni A, Marcon M, Bolgan I, Pellizzari M, Dionisio LD. Systolic blood pressure contributes to intracerebral haemorrhage after thrombolysis for ischemic stroke. J Neurol Sci 2010;297:52-4.

19. Chalela JA, Dunn B, Todd JW, Warach S. Induced hypertension improves cerebral blood flow in acute ischemic stroke. Neurology 2005;64:1979.

20. Kasner SE. Clinical interpretation and use of stroke scales. Lancet Neurol 2006;5:603-12.

21. Weimar C, Konig IR, Kraywinkel K, Ziegler A, Diener HC; German Stroke Study Collaboration. Age and National Institutes of Health Stroke Scale Score within 6 hours after onset are accurate predictors of outcome after cerebral ischemia: development and external validation of prognostic models. Stroke 2004;35:158-62.

22. Sylaja PN, Cote R, Buchan AM, Hill MD; Canadian Alteplase for Stroke Effectiveness Study (CASES) Investigators. Thrombolysis in patients older than 80 years with acute ischaemic stroke: Canadian Alteplase for Stroke Effectiveness Study. J Neurol Neurosurg Psychiatry 2006;77:826-9. 